\title{
Optical Quenching of Magnetic Vortex Visualized In Situ by Lorentz Electron Microscopy
}

\author{
Xuewen $\mathrm{Fu}^{1}$, Shawn D. Pollard ${ }^{2}$, Bin Chen ${ }^{3}$, Byung-Kuk Yoo ${ }^{4}$, Hyunsoo Yang ${ }^{2}$, Yimei Zhu ${ }^{1}$ \\ 1. Condensed Matter Physics and Material Science Department, Brookhaven National Laboratory, \\ Upton, USA. \\ 2. Department of Electrical and Computer Engineering, National University of Singapore, Singapore. \\ 3. Center for Ultrafast Science and Technology, School of Chemistry and Chemical Engineering, \\ Collaborative Innovation Center of IFSA, Shanghai Jiao Tong University, Shanghai, China. \\ 4. Physical Biology Center for Ultrafast Science and Technology, Arthur Amos Noyes Laboratory of \\ Chemical Physics, California Institute of Technology, Pasadena, USA.
}

Magnetic vortex [1, 2] is one of the fundamental spin configurations occurring in thin micro/nanometersized ferromagnetic disks due to the confinement of spins imposed by geometrical restrictions [2, 3]. Understanding the fundamental dynamics of this topological vortex under external perturbations is vital to the applications of magnetic vortex based information processing and spintronic devices. All previous studies have focused on magnetic vortex core switching by external magnetic fields, spin-polarized currents, or spin waves, which have largely prohibited the investigation of novel spin configurations that could emerge from the nominal ground states in ferromagnetic disks and their underlying dynamics.

In this work, we report the in situ visualization of femtosecond (fs) laser quenching induced magnetic vortex change in different symmetric ferromagnetic Permalloy (Py) disks by Lorentz phase imaging in a transmission electron microscope (TEM), as schematically show in Fig. 1. Under the fs-laser pulse excitation above a threshold fluence of about $10 \mathrm{~mJ} / \mathrm{cm}^{2}$, besides the switching of magnetic vortex chirality and polarity, we observed a plenitude of complex magnetic structures with distinct occurrence frequencies. The typical magnetic structure results by fs-laser quenching on a circular and square Py disk are presented in the top and bottom panels of Fig. 2, respectively. Most of these complex magnetic structures have never been observed by magnetic field or current assisted switching. These complex magnetic structures consist of a number of newly created topological magnetic defects (vortex and antivortex) strictly conserving the topological invariant of winding number, demonstrating the direct impact of topological invariant on the magnetization dynamics in ferromagnetic Py disks. Besides that, their spin configurations show mirror or rotation symmetry due to the geometrical confinement of the disks. Combined micromagnetic simulations with the experimental observations reveal the underlying magnetization dynamics and formation mechanism of the optical quenching induced complex magnetic structures. Their distinct occurrence frequencies are pertinent to their energetic and pinning effects at the disk edge. Based on these findings, we further proposed a paradigm of optical-assisted fast switching of vortex cores for the control of magnetic vortex based information recording and spintronic devices [4].

\section{References:}

[1] T Shinjo et al, Science, 289 (2000), p. 930.

[2] A Wachowiak, et al, Science, 298 (2002), p. 577.

[3] K Chou et al, Appl. Phys. Lett., 90 (2007), p. 202505.

[4] The authors acknowledge funding the U.S. Department of Energy, Office of Basic Energy Sciences, Materials Science and Engineering Divisions of the under Contract No. DESC0012704. 


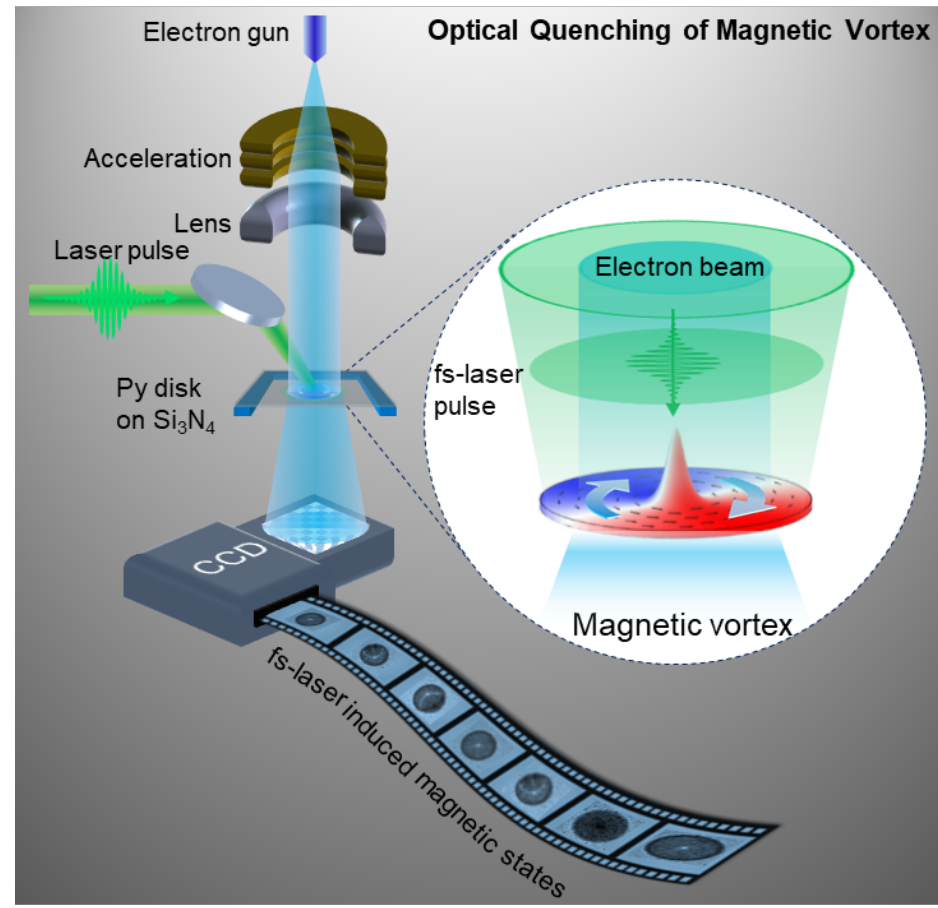

Figure 1. Sketch of in situ imaging fs-laser pulse quenching induced change of spin configuration in a ferromagnetic Py micro disk by TEM operated in Lorentz phase mode. The green fs-laser pulse (520 $\mathrm{nm}, 350 \mathrm{fs}$ pulse duration) for excitation is focused to about $40 \mu \mathrm{m}$ on the sample.

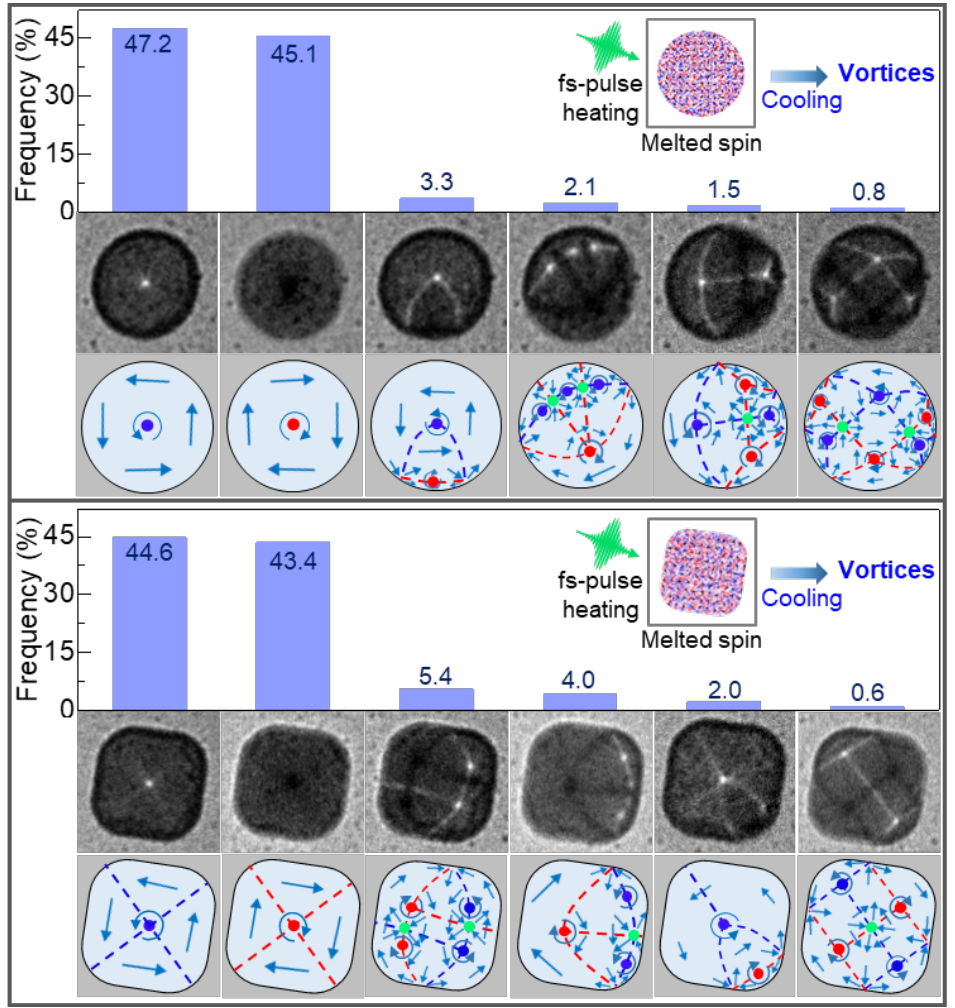

Figure 2. Frequency distribution of the fs-laser pulse (laser fluence of $12 \mathrm{~mJ} / \mathrm{cm}^{2}$ ) induced spin configurations in circular (top panel, with diameter of $3 \mu \mathrm{m}$ ) and square (bottom panel, with edge length of $1.7 \mu \mathrm{m})$ Py disks, respectively. 